\title{
Peste des petits ruminants - crucial challenges for the successful disease eradication
}

\author{
(1)WIES $Ł A W$ NIEDBALSKI, DANDRZEJ FITZNER, \\ KRZYSZTOF BULENGER, @ANDRZEJ KĘSY
}

\begin{abstract}
Department of Foot and Mouth Disease, National Veterinary Research Institute, Wodna 7, 98-220 Zduńska Wola, Poland
\end{abstract}
\section{Niedbalski W., Fitzner A., Bulenger K., Kęsy A. \\ Peste des petits ruminants - crucial challenges for the successful disease eradication \\ Summary}

Peste des petits ruminants (PPR) is a highly infectious and economically important, viral disease of small ruminants caused by the peste des petits ruminants virus (PPRV), which belongs to the genus Morbilivirus in the family Paramyxoviridae. PPR control is mostly achieved through vaccination and/or slaughter of susceptible animals coupled with clinical or laboratory-based diagnosis. The control and eventual eradication of PPR is now one of the top priorities for the Food and Agriculture Organization (FAO) and the World Organization for Animal Health (OIE). In April 2015, the international community agreed on a global strategy for PPR eradication, setting 2030 as a target date for the elimination of the disease. There is a strong and lasting international consensus to eradicate PPR in order to protect the livelihoods of the world's poorest populations. There are several crucial challenges to the eradication campaign programme: understanding small ruminant production, facilitating research to support the eradication campaign, improvement of laboratory diagnostics, optimizing vaccine delivery and novel vaccines, improving epidemiological understanding of the virus, defining infection of wildlife and other species, developing better control and animal movement, heightening serological monitoring, understanding socio-economic impact, and garnering funding and political actions.

Keywords: peste des petits ruminants, control, eradication strategy, challenges

Peste des petits ruminants (PPR) is a highly contagious viral disease of both domestic (goats and sheep) and wild small ruminants, such as springbuck, gazelles and impala (17). It is caused by the PPR virus (PPRV), a member of the genus Morbilivirus of the family Paramyxoviridae and is clinically and pathologically close to rinderpest (RP) and human measles (MV) (4). PPR was first recorded in early 1942 in the Ivory Coast, West Africa (12). In the next years the disease has extended its distribution in other parts of the world and is now circulating throughout Northern, Eastern and West Africa, Asia and Eurasia, the Indian subcontinent, and the Middle East $(4,8)$. It is spreading globally, with its emergence notably reported in China, Mongolia, the Indian subcontinent, Pakistan, Afghanistan, Iran, Iraq, Saudi Arabia and Turkey (8), and recently within the European Union in Bulgaria where PPR outbreak was reported on $24^{\text {th }}$ June 2018 in the village of Voden, Bolyarovo municipality of Yambol region, on the border with the Thrace region of Turkey. In a flock of 540 sheep and goats, two animals tested positive and one died, with the disease confirmed by the EU PPR Reference Laboratory CIRAD-BIOS-UMR - 117,
Montpellier, France (3, http://www.oie.int/wahis_2/ public/wahid.php/Wahidhome/Home). The analysis of PPR outbreaks in recent years helps reveal the latest epidemiological situation of PPR globally and clarify the effects of PPR control in various countries. The total number of PPR outbreaks from 2015 to 2019 (latest data available) in the world is shown in Figure 1, using the World Animal Health Information System (WAHIS). During this period a total of 12757 PPR outbreaks were reported to the OIE by 59 countries, out of 66 recorded to be infected. Among them, 9582 outbreaks were in Asia and the Middle East, 3166 outbreaks were in Africa and 9 PPR outbreaks were in Europe (solely in Bulgaria). In 2015, the number of outbreaks worldwide was 3688, from 2016 to 2018, there were 2858,2405 , and 2588 outbreaks respectively (https://wahis.oie.int/\#/home). In 2019, the number of reported PPR outbreaks showed a marked decrease to 1218 . Since 2015, the number of PPR outbreaks worldwide has been decreasing, and the number of outbreaks in Cameroon, Afghanistan, Kuwait, and Oman has decreased year by year. Currently, 21 of 66 countries classified as infected have had no reported 


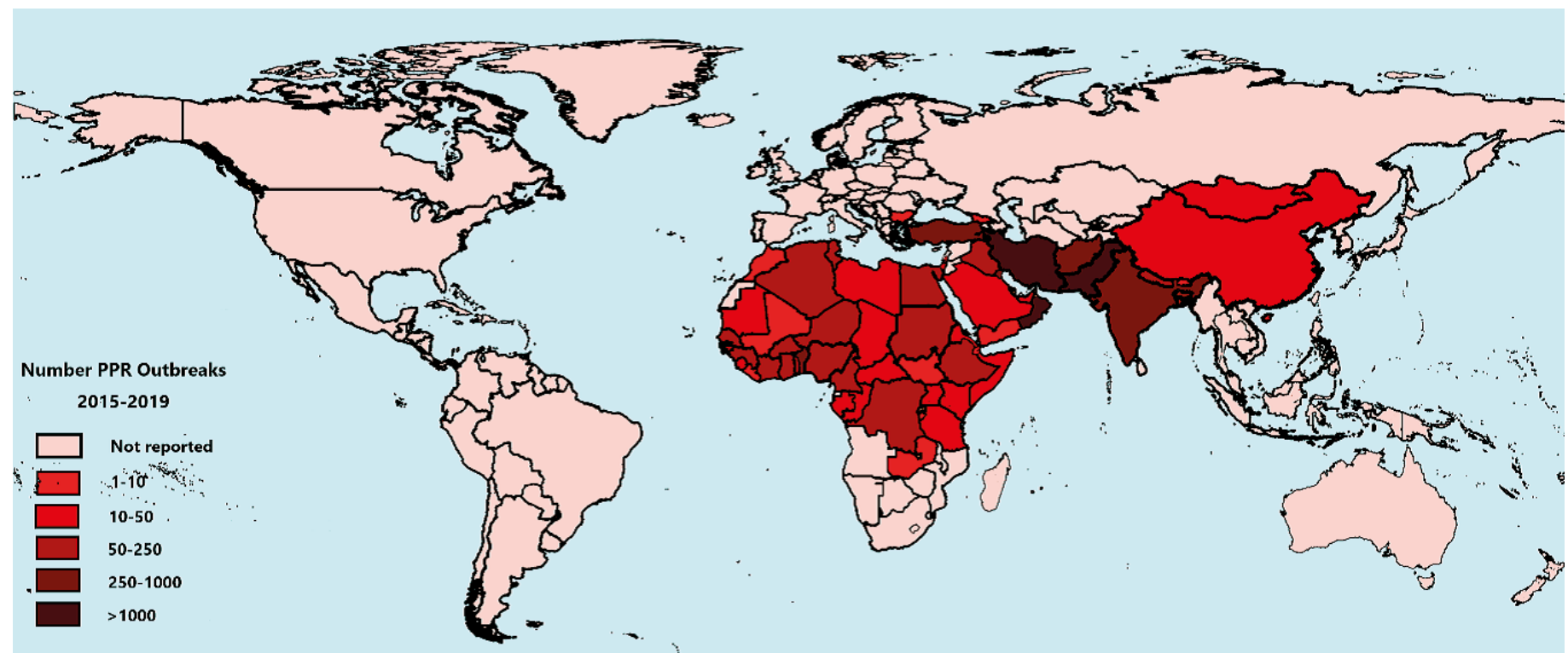

Fig. 1. The worldwide distribution of PPR showing the total number of outbreaks from 2015 to 2019 (according to WAHIS)

PPR outbreaks for more than 24 months, and 10 of these have had no PPR outbreaks between 2015 and 2019, namely Angola, Bahrain, Jordan, Kazakhstan, Kyrgyzstan, Lebanon, Qatar, Syrian Arab Republic, Tajikistan, and Uzbekistan. Notably, the number of PPR outbreaks in many countries is high: 22 countries suffered more than 100 outbreaks between 2015 and 2019, including 15 in Africa and 7 in Asia. Of these, more than 400 PPR outbreaks have been reported in 5 countries, namely Benin (480), Afghanistan (824), Iran (3710), Kuwait (761), and Turkey (402). From 2015 to 2019, PPR outbreaks in these five countries accounted for $48.4 \%$ of all global outbreaks. In addition, there has been an increase in reported outbreaks in 2018 and 2019 in Algeria, Ethiopia, and Guinea.

PPR causes huge losses to the economy and income of developing countries, representing a threat to the primary source of livelihoods for above 300 million families who rely on small ruminants. PPR has direct loss such as mortality and indirect loss such the cost of treatment and reduction of animal body condition and has negative impact on export earnings. Both direct and indirect losses of the economy in the whole world due to PPR was estimated to be about USD 3 billion per year during 2012-2017 $(11,15)$. As a response, the Food and Agriculture Organization of the United Nations (FAO) and the World Organization for Animal Health (OIE) endorsed the Global Strategy for the Control and Eradication of PPR (PPR GCES), and launched the PPR Global Eradication Programme (PPR GEP), to eradicate PPRV by 2030. During the meeting of FAO and OIE experts in April of 2015 in Abidjan, Cote d'Ivoire the role of epidemiology, vaccination, and the capacity of national veterinary services to manage an eradication program has been discussed. Management of disease eradication programs is an adaptive and dynamic process that builds on available knowledge, new learning and new technologies (8).
The eradication of PPR by 2030 is a huge effort for countries where the disease occurs. So the eradication effort will involve a combined approach of strengthening countries' veterinary health services and systems for disease surveillance, vaccination campaigns, and awareness raising and capacity building. Strategic control and eradication must be designated to reduce and prevent further spread of PPR and increase the health of the animals as well as the control infected zones from PPR and prevent the spread to disease free zones.

\section{Factors that support and limit PPR eradication}

A number of factors support PPR eradication, such as: 1) PPRV is fragile outside the host, as its lipid bilayer envelope is rapidly destroyed by heat and sunlight (12). It is mainly transmitted by direct contact, local aerosol spread from coughing, or contaminated feed or water, but only to animals within close proximity; 2) The vaccine against PPR protects against all lineages, it provides long lasting protection (at least 3-5 years, but probably life-long), it is safe, in that it has not reverted to virulence and does not cause abortion, and it is also widely available and quality controlled (14); 3) There is not a prolonged carrier state after PPRV infection, and there are no known reservoirs outside domestic small ruminants; 4) The clinical signs of PPR are easily detected by herders. In previously free populations, the disease takes an epidemic form, with high morbidity and mortality and acute clinical expression, making clinical detection relatively reliable. Besides, there are good laboratory and field-based diagnostic tests available.

However, there are a number of potential limitations of disease eradication, for instance: a) for effective PPR eradication programmes worldwide (PPR-GEP) it is imperative to prove the role of large ruminants and unusual hosts to better elucidate disease epidemiology, particularly in disease-enzootic regions (19); 
b) PPR is extremely widespread and endemic in many regions with under-resourced veterinary services; c) population dynamics is important constraints, as small ruminants have a high population turnover, resulting in the rapid introduction of naive animals into vaccinated population (14).

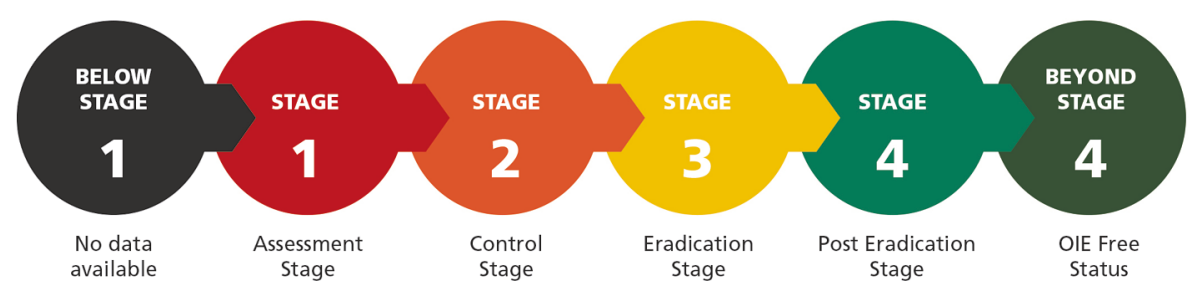

Fig. 2. The stages of PPR GEP Local animal density within flocks is high, facilitating rapid within-flock/herd spread; d) clinical expression of PPR varies with species and breeds, and the signs are not specific, making a definitive clinical diagnosis difficult or even impossible. In endemic areas, PPRV may circulate with little clinical expression; e) the costs of eradication should be assessed: while the total cost of PPR is high, the value of individual animals (relative to cattle, for example) is much lower. This, coupled with short lifespan, means that the proportional cost of vaccination is higher than was the case for rinderpest.

\section{The stages of PPR eradication}

The PPR-GEP is a multi-country, multi-stage process that will decrease epidemiological risk levels and increase prevention and control. This programme promotes a stepwise approach to eradication on four stages (Fig. 2). The four stages set out involve assessment, control, eradication and maintenance of PPR-free status. This ranges from stage 1 (where the epidemiological situation is being assessed), to stage 4 (when the country provides evidence that there is no virus circulation either at the zone or national level, and is ready to apply for OIE-official PPR-free status). Control activities, including vaccination, are implemented in stage 2 while stage 3 corresponds directly to PPR eradication. Of note, to enter stage 4 vaccination must be suspended in order to facilitate epidemiological monitoring of PPR. Implementation requires the concerted delivery of preparedness plans, capacity building, improved stakeholder awareness and engagement, as well as the establishment of appropriate legal frameworks $(8,9)$. Regardless of the stage in which a country initially places itself, it will be supported to achieve the capacity it needs for the five key elements of PPR prevention and control: diagnostic system, surveillance system, prevention and control system, legal framework, and stakeholder involvement. Putting these five technical elements in place will enable any country to move with confidence to the next stage of control and eradication.

\section{The fundamental challenges to the PPR eradication campaign programme}

There are several crucial challenges to the eradication campaign programme:

1. Small ruminant production. At the national level, small ruminants lobbies often have limited access to decision makers or resources partners, reducing the attention given to PPR (and small ruminant health in

general). Another challenge facing small ruminant production is the short cycle nature of production or annual turnover rate of $30 \%$ to be considered regarding the vaccination programme. This implies a very different cost benefit picture for veterinary services and household unit in relation to investments in vaccines and other animal health inputs. It is therefore important for any eradication strategy to engage the small ruminant owners in improving their own system and enhancing private sector service delivery channels.

2. Studies on the facilitation of eradication. This may include research on: socio-economic analysis, epidemiological studies, e.g., on disease transmission chains, identification of small ruminant populations acting as reservoirs and modelling of this, identifying the genetic determinants of virulence, diagnostic and surveillance methodology, molecular epidemiology, filter paper sampling for serology, rapid field tests and, optionally, novel vaccines (marked and recombinant, e.g., vaccines able to distinguish vaccinated from naturally infected [DIVA] animals). Studies in epidemiology will help in informing vaccination strategies. Few of these studies will assist early diagnosis at the field level or transportation of specimens to the absence of cold chain facilities. The research for diagnostic tests for understanding their suitability to be used for PPR investigation in non-typical animal hosts, and if needed, development of new tests that are fit for the purpose. Research can also assist for differential diagnostics for post-vaccination monitoring/reassurance. The existing vaccines have already been developed in thermostable forms and production has started. Other areas of research worth highlighting include an apparent variation in disease severity, which has been observed between different breeds of goats (7). Further studies are required to define the susceptibility and to examine the variation extents to specific features such as the duration of viral shedding in infected animals, factors which will contribute to the dynamics of PPR transmission. Another example would be developing a better understanding of how small ruminants are bred, raised, moved, traded and slaughtered in PPR endemic countries. This will enable a broader appreciation of the lifestyle of the livestock keepers who own and depend on these animals. The acquired knowledge will help to develop effective strategies for PPR vaccination campaigns.

3. Laboratory diagnosis of PPR in the eradication campaign. The serological, virological and molecular diagnostic methods of PPR are essential components 
of the four stages of the PPR global eradication strategy, from assessment to the post-eradication phase. However not all routinely used diagnostic techniques may be suitable for all stages of PPR control and eradication. For instance, diagnostics such as ELISA could be used for mass screening of clinical and serum samples, whereas immuno-chromatographic tests can be used at the field levels (pen-side tests). Work to define minimal performance characteristics of these diagnostic assays and to establish bench marking procedures for diagnostic networks is needed. Assays with higher sensitivity, such as competitive ELISA, qRT-PCR and Loop Mediated Isothermal Amplification (LAMP) are clearly important for early diagnosis of PPR and also, theoretically, during the late stages of eradication or when sampling atypical hosts, e.g., wildlife with suspected infection (13). During the late stages of any control programme, suspected outbreaks will also have to be reconfirmed using multiple laboratory tests (20). Defining when and how to implement an appropriate range of laboratory diagnostic tests remains a multifactorial challenge, depending on assay availability, training needs and deficits, various cost-benefit analyses and the correct validation of the assays used.

4. PPR vaccines. Currently, the PPR control and eradication strategy includes administration of a liveattenuated vaccine, which following one inoculation provides lifelong immunity to all PPRV lineages as well as detectable antibodies. However, currently, a lot of vaccines do not reach sufficient levels of immunity to interrupt transmission. The most challenging practical issues are related to the delivery of vaccines to small ruminants at sufficient intensity to generate herd immunity levels that are able to prevent the re-introduction of disease. Within the pastoral area, there is often a lack of harmonisation of vaccination campaigns at the epizootic level. This is also due to the lack of simultaneous funding of PPR activities in neighbouring countries. There is a need for countries to define precise eradication strategies and implement them in a dynamic manner. The concept of using repeat pulses of extremely efficient vaccination, separated by short time intervals, in defined areas could be used. This technique might be explored further by epidemiological modelling with the hope that it can break virus transmission in endemic areas, when supported by good clinical disease surveillance. It was found that integrating the supply chain of PPR vaccine with other veterinary or health commodities could reduce costs, as well as increase uptake (2). Separately, maintenance of an effective cold chain for this vaccine has also proven difficult in subtropical countries. Application of a thermotolerant live-attenuated conventional or recombinant vaccine may represent the best way to avoid cold chain-associated problems in these areas. However, it must also be noted that although the standard PPR vaccines are cheap and effective, there is a need, especially in developing countries, for vaccines that support broader sustainability in small ruminant production. To this end, there have been significant advances in designing multivalent small ruminant virus vaccines that can also contribute to PPR eradication. Additional field application of these multivalent vaccines to control common small ruminant pathogens, including PPRV, would indeed help to enhance poverty alleviation. While risk-based vaccination of sheep and goats in endemic countries might be a pragmatic approach to control PPR in the first phase of disease eradication, the development of a marker vaccine with a robust companion test may help with serological surveillance in the future. Other possibilities include the development of a vaccine capable of DIVA. Emphasis in this area should also be placed on development models for these vaccines, as well as their applicability in various hosts, to ensure they are utilised to their maximum potential. The development of recombinant PPR vaccines and virus-like particles that are relatively more thermo-stable and have DIVA capability through the use of companion diagnostic tests to reduce cost and time for effective control and eradication of disease. In conclusion, identifying and targeting high-risk populations through vaccination campaigns informed by the estimation of context-specific PPRV transmission levels would not only reduce the cost of PPR eradication, but by setting more achievable vaccination coverage also increase the likelihood of success.

5. Epidemiological understanding of PPRV. Epidemiological research is required to better understand the dynamics of PPR transmission, in particular its spread and infectivity and the different roles of wildlife and livestock species, production systems, ecosystems and viral lineages in this process. The overall goal is to identify critical control points, and optimal methods for intervention at these points, to support effective management of eradication. The results of these studies will likely be the main driver for decisions about what levels of control might practically be achieved as well as the feasibility of timely eradication according to the stages of the PPR-GEP. At a smaller scale, RP eradication highlighted the importance of local stakeholder involvement: e.g., the direct involvement of livestock keepers in the process of tracing disease as being critically important in disease monitoring. The implementation of these local networks combined with a continued global appreciation of PPRV epidemiology will be key to the long-term sustainability of the PPR eradication campaign. Other epidemiological relevant issues such as the characterization of viral propagation rates, the decay rate of maternal antibodies, and agespecific infection and case fatality rates, are also early priorities for avoiding PPR-re-emergence in disease free areas.

6. PPR in domestic livestock of other species and wildlife. There are convincing reports demonstrating the ability of PPRV to cross the species barrier. Indeed, PPRV can infect animal species other than 
small ruminants such as cattle, buffalo, dogs, Asiatic lion and pigs $(1,19,21,22)$. It is unclear whether these infections are relevant from an epidemiological and eradication perspective; however, it is essential to fully understand the role of wildlife small ruminants in the spread and potential maintenance of PPRV in the environment in order to be able to initiate successful control strategies. The role of wildlife in PPR epidemiology can be presented on the example of a PPR outbreak in Mongolia. In December 2016 the disease was diagnosed in several wildlife populations in the east of the country, including saiga antelope, ibex and gazelle, with more than $80 \%$ mortality (10). Studies in camel populations, kept together with small ruminants in Kenya, established that camels in the study area suffer with PPR manifesting clinical signs that are mainly characterized by inappetence, loss of body condition, and general weakness, leading to diarrhea, conjunctivitis, and ocular nasal discharges preceding death. These clinical signs are similar to those observed in small ruminants with slight variations of manifestations such as keratoconjunctivitis as well as oedema of the ventral surface of the abdomen. This shows that camels could be involved in the epidemiology of PPR in the region and that PPRV could be involved in epidemics of disease in camels $(16,18)$. Therefore an important first step is to ensure that the currently available tests for serological diagnosis of PPRV are validated in serum samples from these animal species, e.g., camels, saiga and ibex. With specific reference to the PPRV eradication campaign, the significance of these infections as a whole should be carefully evaluated, as they may not significantly affect the ultimate success of the programme.

7. Movement control and communication. The control of animal movement is integral to most infectious disease control and eradication programmes. Strict movement control can be counter-productive because it can actually stimulate unnecessary or illegal movement of animals in order to bypass quarantines and restriction orders. Movement control must be tempered by the experience of local animal health teams who are better equipped to judge the behaviour of local owners when faced with such restrictions. Better communication is also essential for developing and influencing people's attitudes, behaviour and decisions concerning the control of PPR. It is also essential to learn and share knowledge about the attitudes of livestock owners and other key stakeholders. Social science methodologies could be used across the whole eradication campaign to maintain the link between local stakeholders and the eradication team at the regional level or to explore potential options such as making herders contribute or not to the vaccination. The absence of communication at this level is another challenge to be addressed.

8. Serological monitoring and the demonstration of absence of PPR. Serological monitoring should be used to assess the success rates of vaccination campaigns or to invigilate the effectiveness of individual vaccination teams. However, in reality it may be useful if immediate revaccination can be carried out, which is not often possible. The case for detailed serological monitoring may have to be made on a case-by-case basis, depending on a cost-benefit analysis and whether the resultant data can realistically contribute to improve long-term planning. In at risk areas that have never declared the disease or when a country has stopped the vaccination for a few years, serology could be carried out to demonstrate the absence of PPRV. In the absence of DIVA and the current situation in several countries, the challenge is the interpretation of positive samples. Careful surveillance strategy taking in to account the vaccination history should be considered.

9. Socio-economic impact. The economy-wide considerations of emerging diseases are crucial in implementing and funding control and eradication strategies. For PPR a well-planned cost-benefit analysis of the disease, comparing policies and responses that include both the direct and indirect impacts associated with PPR are needed to better understand the impact PPR in all settings. That being said, the annual global impact of PPR has been estimated at between USD 1.4 billion and USD 2.1 billion, with cost-benefit studies also carried out in different countries; estimating losses which clearly justify both national and global PPR eradication programmes being pursued (9).

10. Funding and political actions. Many of the countries where PPR is now endemic will need significant support for operational costs, training and meetings in order to properly implement PPR GEP. Even if livestock owners themselves contribute more towards the costs of vaccination, there will still be a requirement at the regional and global level for international funding to provide technical and coordination costs, as well as member state support $(6,9)$. The PPR GEP budget for 2017-2021, was estimated at USD 996 million. The majority of the funding for PPR GEP relies on resources at a country level, in particular national budgets (9). It was shown that trade routes, refugee camps and areas of animal crowding during droughts increase PPR incidence as well as the socio-economic impact. These should be targeted for interventions, monitoring and surveillance as part of PPR control programmes (23). However, global funding is needed for some catalytic components of the programme, including activities that support the effective mobilization of additional national and regional resources. It was estimated that of the approximately USD 1 billion needed for the first PPR GEP phase (2017-2021), USD 656 million has been secured through national budgets and resource partners. The critical financial gap was therefore estimated at USD 340 million $(9,11)$. It is worth highlighting that more than $70 \%$ of the PPR GEP budget is allocated for the vaccination of 1.5 billion sheep and goats (this includes costs for 
vaccine procurement, logistics and post vaccination evaluation). However, it should be mentioned that of the funding already secured, only a small portion of these resources is dedicated to PPR control and eradication.

In conclusion it can be stated that the PPR eradication efforts will involve a combined approach of strengthening countries veterinary raising and capacity building. It will require the support of many institutions like national veterinary services of respective countries, research and development organizations, vaccine manufacturing companies, etc. The final eradication could be achieved rapidly if the seat of infection was known and if appropriate national and international interventions were aimed at ending virus transmission. For such international cooperation to be forthcoming once more, the understanding, tools and experiences now on offer suggest that PPR could be controlled and eradicated far more rapidly than RP.

\section{Reference}

1. Abraham G., Sintayehu A., Libeau G., Albina E., Roger F., Laekemariam Y., Abayneh D., Awoke K. M.: Antibody sero-prevalences against peste des petits ruminants (PPR) virus in camels, cattle, goats and sheep in Ethiopia. Prev. Vet. Med. 2005, 70, 51-57.

2. Acosta D., Hedrickx S., McKune S.: The livestock vaccine supply chain: why it matters and how it can help eradicate peste des petits ruminats, based on findings in Karamoja, Uganda. Vaccine 2019, 37, 6285-6290.

3. Altan E., Parida S., Mahapatra M., Turan N., Yilmaz H.: Molecular characterization of peste des petits ruminants viruses in the Marmara region of Turkey. Transbound. Emerg. Dis. 2019, 66, 865-872.

4. Banyard A. C., Parida S., Batten C., Oura C., Kwiatek O., Libeau G.: Global distribution of peste des petits ruminants virus and prospects for improved diagnosis and control. J. Gen. Virol. 2010, 91, 2885-2897.

5. Baron M. D., Diallo A., Lancelot R., Libeau G.: Peste des petits ruminants virus. Adv. Virus Res. 2016, 95, 1-42.

6. Baron M. D., Parida S., Oura C. A. L.: Peste des petits ruminants: a suitable candidate for eradication? Vet. Rec. 2011, 169, 16-21.

7. Diop M., Sarr J., Libeau G.: Evaluation of novel diagnostic tools for peste des petits ruminants virus in naturally infected goat herds. Epidemiol. Infect. $2005,133,711-717$

8. FAO and OIE International Conference for PPR: Control and eradication of peste des petits ruminants (PPR), Global strategy for the control and eradication of PPR, Abidjan, Cote D'Ivoire, 31 March-2 April 2015 (www.fao.org/3/ a-i4460e.pdf).

9. FAO/OIE. PPR Global Eradication Programme 2017-2021. 2016. (http://www. fao.org/3/a-i6316e.pdf).

10. Fine A. E., Pruvot M., Benfield C. T. O., Caron A., Cattoli G., Chardonnet P., Dioli M., Dulu T., Gilbert M., Kock R., Lubroth J., Mariner J. C., Ostrowski S., Parida S., Fereidouni S., Shiilegdamba E., Sleeman J. M., Schulz C., Soula J. J., Van der Stede Y., Tekola B. G., Walzer C., Zuther S., Njeumi F., Meeting Participants.: Eradication of peste des petits ruminats virus and the wildlifelivestock interface. Front. Vet. Sci. 2020, 7, 50, doi: 10.3389/fvets.2020.00050. eCollection 2020.

11. Jones B. A., Rich K. M., Mariner J. C., Anderson J., Jeggo M., Thevasagayam S. Cai Y., Peters A. R., Roeder P. L.: The economic impact of eradicating peste des petits ruminants: a benefit-cost analysis. PLoS One 2016, 11, e149982, doi: 10.1371/journal.pone.0149982. eCollection 2016.

12. Lefevre P. C., Diallo A.: Peste des petits ruminants. Rev. Sci. Tech. 1990, 9 , 935-981

13. Mahapatra M., Howson E., Fowler V., Batten C., Flannery J., Selvaraj M., Parida S.: Rapid detection of peste des petits ruminants virus (PPRV) nucleic acid using a novel low-cost reverse transcription loop-mediated isothermal amplification (RT-LAMP) assay for future use in nascent PPR eradication programme. Viruses 2019, 11, 699, doi: 10.3390/v11080699.

14. Mariner J. C., Jones B. A., Rich K. M., Thevasagayam S., Anderson J., Jeggo M., Cai Y., Peters A. R., Roeder P. L.: The opportunity to eradicate peste des petits ruminants. J. Immunol. 2016, 196, 3499-3506.
15. Nour H. S. H.: Challenges and opportunities for global eradication of peste des petits ruminants (PPR). J. Trop. Dis. 2020, 8, 349, doi: 10.35248/2329891X.20.8.349.

16. Omani R. N., Gitao G. C., Gachohi J., Gathumbi P. K., Bwihangane B. A., Abbey K., Chemweno V. J.: Peste des petits ruminants (PPR) in dromadery camels and smal ruminants in Mandera and Wajir counties of Kenya. Adv. Virol. 2019, 4028720, doi: 10.1155/2019/4028720.

17. Parida S., Muniraju M., Mahapatra M., Muthuchelvan D., Buczkowski H., Banyard A. C.: Peste des petits ruminants. Vet. Microbiol. 2015, 181, 90-106.

18. Pruvot M., Fine A. E., Hollinger $C$., Strindberg S., Damdinjav B. Buuveibaatar B., Chimeddorj B., Bayandonoi G., Khishgee B., Sandag B., Narmandakh J., Jargalsaikhan T., Bataa B., McAloose D., Shatar M., Basan G., Mahapatra M., Selvaraj M., Parida S., Njeumi F., Kock R., Shiilegdamba E.: Outbreaks of peste des petits ruminants among critically endangered Mongolian Saiga and other wild ungulates, Mongolia 2016-2017. Emerg. Infect. Dis. 2020, 26, 51-62.

19. Rahman A. U., Dhama K., Ali Q., Hussain I., Oneeb M., Chaudhary U. Wensman J. J., Shabbir M. Z.: Peste des petits ruminats in large ruminants, camels and unusual hosts. Vet. Q. 2020, 40, 35-42.

20. Roger F., Diallo A., Yigezu L. M., Hurard C., Libeau G., Mebraru G. Y., Faye B.: Investigation of a new pathological condition in camels in Ethiopia. J. Camel Pract. Res. 2000, 7, 163-166.

21. Santhamani R., Singh R. P., Njeumi F.: Peste des petits ruminants diagnosis and diagnostic tools at a glance: Perspectives on global control and eradication. Arch. Virol. 2016, 161, 2953-2967.

22. Schulz C., Fast C., Schlottau K., Hoffmann B., Beer M.: Neglected hosts of small ruminat Morbilivirus. Transpound. Emerg. Dis. 2018, 24, 2334-2337.

23. Spiegel K. A., Havas K. A.: The socioeconomic factors surrounding the initial emergence of peste des petits ruminants in Kenya, Uganda, and Tanzania from 2006 through 2008. Transbound Emerg. Dis. 2019, 66, 627-633.

Corresponding author: Wiesław Niedbalski, PhD, DSc, Wodna 7, 98-220

Zduńska Wola, Poland; e-mail: wieslaw.niedbalski@piwzp.pl 